\title{
PARÁLISIS DOLOROSA INCOMPLETA DEL III NERVIO CAUSADA POR UN ANEURISMA DE LA CARÓTIDA INTERNA-COMUNICANTE POSTERIOR
}

\author{
PAINFUL INCOMPLETE THIRD-NERVE PALSY CAUSED BY \\ AN INTERNAL CAROTID-COMMUNICATING POSTERIOR \\ ARTERY ANEURYSM
}

\author{
PÉREZ-FLORES MI ${ }^{1}$, VELASCO-CASARES M², LORENZO-CARRERO J ${ }^{3}$
}

\begin{abstract}
RESUMEN
Caso clínico: Mujer de 38 años que presentó de forma aguda una parálisis incompleta y dolorosa del III par causada por un aneurisma de la carótida interna-comunicante posterior (CI-CP). Describimos el diagnóstico diferencial con el Síndrome Tolosa-Hunt y los resultados tras tratamiento endovascular.

Discusión: La presencia de una parálisis del III par sin afectación pupilar no excluye la presencia de un aneurisma de la CI-CP. La realización de una resonancia nuclear magnética orbitaria y/o angio-tomografía axial computerizada es obligada en estos casos. La rápida recuperación tras tratamiento endovascular es consistente con otros trabajos previos.

Palabras clave: Parálisis dolorosa incompleta del III nervio, Tolosa-Hunt, aneurisma de la carótida interna-comunicante posterior, RNM, angioTAC, embolización.
\end{abstract}

\begin{abstract}
Clinical case: A 38-year-old woman presented acute-onset, painful incomplete third-nerve palsy caused by an internal carotid-posterior communicating artery (IC-PC) aneurysm. We describe the differential diagnosis with Tolosa-Hunt syndrome and the results after endovascular treatment.

Discussion: The presence of pupil-sparing thirdnerve palsy may not exclude the presence of an ICPC aneurysm and orbital magnetic resonance imaging and/or computed tomography imaging must be performed in these cases. The prompt recovery after endovascular treatment is consistent with previous reports (Arch Soc Esp Oftalmol 2009; 84: 43-46).
\end{abstract}

Key words: Painful incomplete third-nerve palsy, carotid-posterior communicating artery aneurysm, Tolosa-Hunt, MRI, CAT, embolization.

\footnotetext{
Recibido: 12/12/07. Aceptado: 10/12/08.

Servicio de Oftalmología. Hospital povisa. Vigo (Pontevedra). España.

1 Licenciada en Medicina.

2 Licenciada en Medicina. Servicio de Radiología.

3 Doctor en Medicina.

Correspondencia:

María Inés Pérez Flores

Servicio de Oftalmología. Hospital POVISA

C/. Salamanca, 5

36211 Vigo (Pontevedra)

España

E-mail: mari-nes@eresmas.net
} 


\section{INTRODUCCIÓN}

Los aneurismas son la causa más frecuente de afectación del III par a nivel subaracnoideo. La forma de presentación clínica puede incluir el comienzo agudo de dolor periocular, ptosis, limitación de la adducción, depresión y elevación del ojo y pupila dilatada (1).

En adultos, una parálisis oculomotora dolorosa y con pupila afectada puede ser el resultado del crecimiento de un aneurisma intracraneal y es un signo de alarma de esa malformación vascular. La ruptura de un aneurisma determina una hemorragia subaracnoidea súbita, con una alta tasa de morbilidad y mortalidad. El diagnóstico neuro-radiológico y el tratamiento endovascular pueden prevenir este catastrófico suceso (2).

\section{CASO CLÍNICO}

Mujer de 38 años que presentó cefalea y dolor periorbitario izquierdo de una semana de evolución y diplopía las últimas 24 horas. La exploración motora reveló una paresia de los músculos recto superior e interno y ptosis moderada en el ojo izquierdo. No hubo otros hallazgos oculares o neurológicos. La resonancia nuclear magnética (RNM) craneal fue normal. Se diagnosticó un síndrome de
Tolosa-Hunt y se inició tratamiento con corticoides. Dos días después, la oftalmoplejía dolorosa persistía y la paciente comenzó a referir disminución de agudeza visual próxima. En la exploración de la paciente se observó midriasis izquierda. Se realizó RNM orbitaria que mostró en T2 un aneurisma de la carótida interna-comunicante posterior (CI-CP) parcialmente trombosado. La angio-tomografía axial computerizada (angio-TAC) mostró un aneurisma CI-CP izquierdo multilobulado de $10 \times 5 \mathrm{~mm}$ (fig. 1). Se realizaron angiografía cerebral y embolización (fig. 2), siendo rellenado completamente el aneurisma con Hydrocoil (MicroVention, Aliso Viejo, CA) Cerecyte ${ }^{\circledR}$ (Micrus, Sunnyvale, CA). El tamaño pupilar se normalizó y la ptosis y la adducción mejoraron completamente dos semanas después de la embolización. Ocho meses tras el tratamiento persistía un ligero déficit en la elevación, sin que la paciente refiriese diplopía.

\section{DISCUSIÓN}

La existencia de parálisis del tercer par secundaria a un aneurisma de la carótida interna-comunicante posterior ha sido previamente descrita (1). Cuando la pupila no está afectada la causa más común de esta parálisis es vascular, con frecuencia diabetes. La lesión está confinada a la parte interna

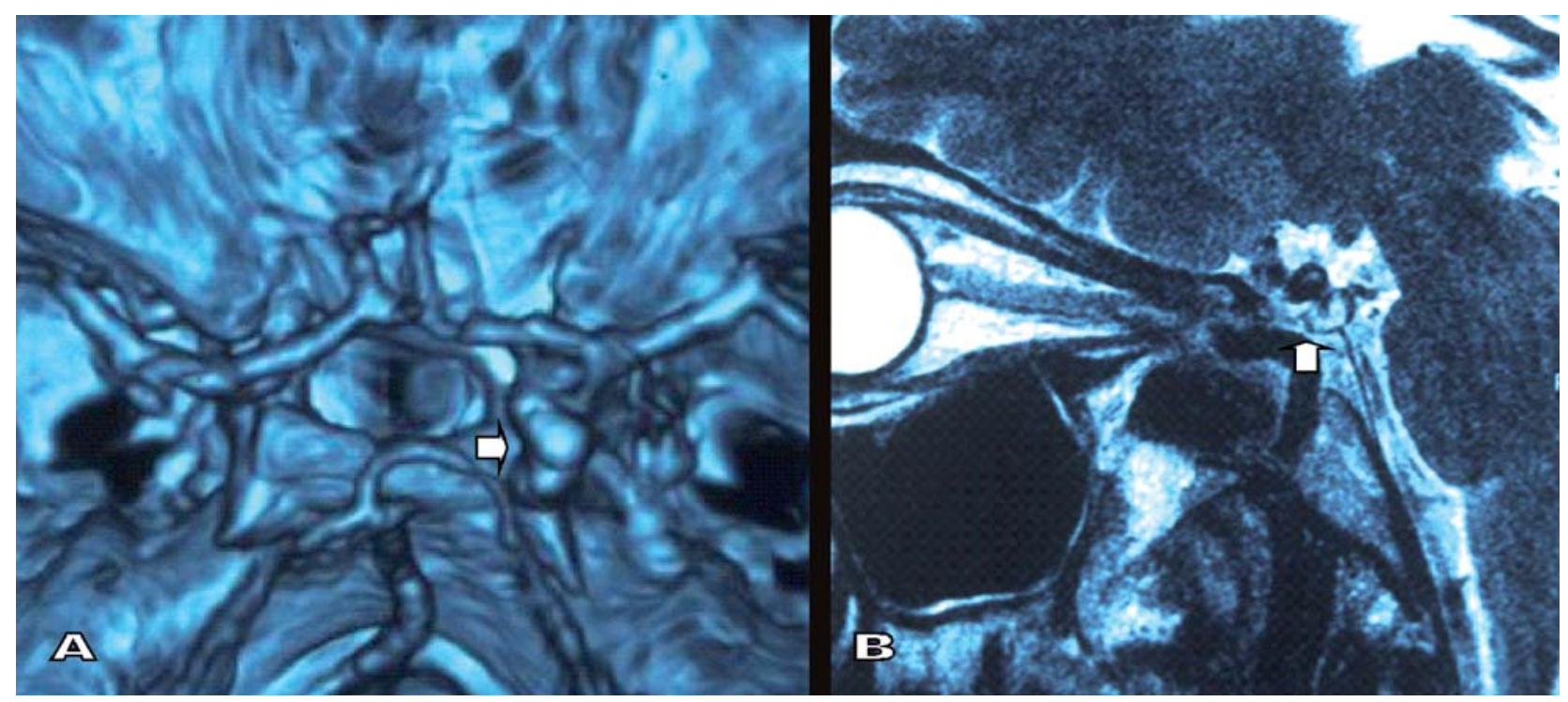

Fig. 1: A: Resonancia nuclear magnética (RNM) T2 (reconstrucción 3D), mostrando el aneurisma de la carótida interna-comunicante posterior (CI-CP) trombosado. B: Aneurisma CI-CP izquierdo demostrado por angio-tomografía axial computerizada. 


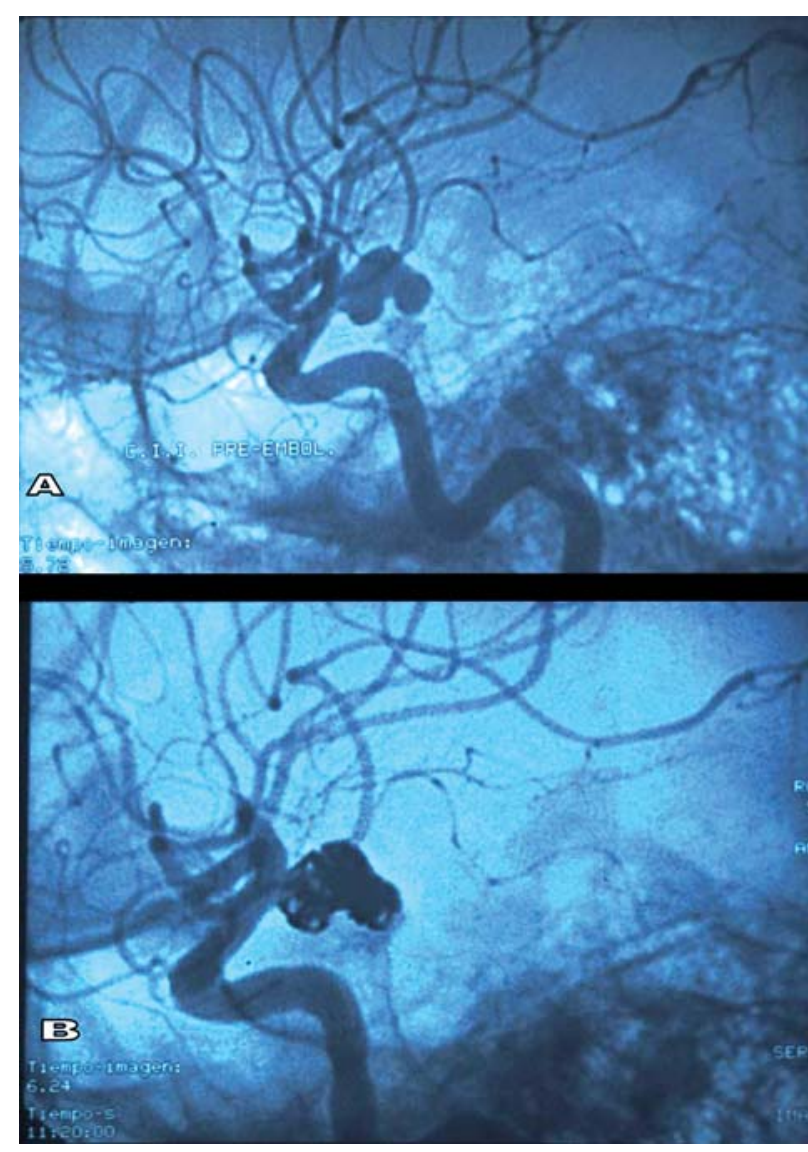

Fig. 2: A: Angiograma carotídeo izquierdo, mostrando el aneurisma intacto en la unión de la carótida interna izquierda y la comunicante posterior. B: Angiograma tras la embolización, mostrando la obliteración completa del aneurisma mediante el Hydrocoil Cerecyte ${ }^{\circledR}$.

del nervio y se respetan las fibras pupilomotoras localizadas en la periferia. Por contraste, cuando la pupila está afectada, la causa más frecuente es un aneurisma que comprime el nervio. Sin embargo, la compresión aislada de la porción inferior del nervio no afecta a las fibras pupilomotoras localizadas en la porción superior. La pupila puede verse respetada inicialmente, pero finalmente se afecta la vía eferente causando midriasis (3).

Un aneurisma CI-CP intacto, asociado o no con una parálisis oculomotora, puede provocar dolor orbitario como síntoma de alarma previo a su ruptura. Dolor fronto-orbitario puede resultar de la distorsión de las fibras que vienen de la división oftálmica del V par, cuando el crecimiento del aneurisma afecta al III par por sí mismo. Trabajos previos confirman que la ausencia de cefalea y/o dolor ocular o la presencia de parálisis del III par sin afecta- ción pupilar, como en nuestro caso, puede no excluir la presencia de un aneurisma CI-CP $(1,3)$.

El síndrome de Tolosa-Hunt es una oftalmoplejía dolorosa caracterizada por dolor unilateral recurrente, parálisis oculomotora ipsilateral y rápida respuesta a los corticoides. Se origina por una inflamación inespecífica del seno cavernoso o el ápex orbitario. La Mantia et al observaron que el III par es el más comúnmente afectado en el síndrome de Tolosa-Hunt y aproximadamente un tercio de los casos presentan RNM normal (5).

Nuestra paciente mostró una parálisis dolorosa incompleta aguda, sin afectación de la pupila inicialmente y RNM craneal normal. En ausencia de otros datos patológicos se diagnosticó de síndrome de Tolosa-Hunt y se inició el tratamiento con corticoides. La RNM orbitaria y la angio-TAC se realizaron tras observar la afectación pupilar tardía y la ausencia de respuesta a al tratamiento, comprobándose así la presencia de un aneurisma CI-CP izquierdo.

Ante una parálisis incompleta del III par sin afectación pupilar debe revisarse al paciente diariamente en la primera semana. Se ha de sospechar la presencia de un aneurisma en pacientes de edades comprendidas entre los 20 y 50 años y sin otros factores vasculares de riesgo. La angiografía cerebral se ha sugerido como método de exploración rutinario en pacientes con parálisis del III par. Sin embargo, dicha prueba puede estar asociada con una tasa de accidente vascular cerebral de 0,6-5\% especialmente en pacientes de edad avanzada y alto riesgo vascular. La angio-TAC y la angio-RNM han sido documentados como métodos diagnósticos en pacientes con parálisis del III nervio, así como su capacidad de detectar aneurismas de un tamaño mayor o igual a $3 \mathrm{~mm}$. Wong et al concluyen que, como primera línea de investigación, la angio-TAC es suficiente para detectar aneurismas compresivos y puede detectar otras lesiones estructurales. Pacientes que tienen angio-TAC de poca calidad o mostrando un aneurisma que necesita embolización, necesitarán una angiografía cerebral (3).

La embolización reduce la pulsatilidad del aneurisma, lo cual puede ser más relevante en la resolución de la parálisis del III par que la liberación anatómica del nervio tras la escisión quirúrgica del aneurisma (4).

La pronta recuperación de la parálisis del III par en nuestro caso, es consistente con los trabajos previamente publicados en los que se sugiere una más rápida resolución de los déficits mediante tratamiento endovascular (2). 


\section{BIBLIOGRAFÍA}

1. Dimopoulos VG, Fountas KN, Feltes CH, Robinson JS, Grigorian AA. Literature review regarding the methodology of assessing third nerve paresis associated with nonruptured posterior communicating artery aneurysms. Neurosurg Rev 2005; 28: 256-260.

2. Inamasu J, Nakamura Y, Saito R, Kuroshima Y, Ohba S, Ichikizaki K. Early resolution of third nerve palsy following endovascular treatment of a posterior communicating artery aneurysm. J Neuroophthalmol 2002; 22:12-14.
3. Wong GK, Boet R, Poon WS, Yu S, Lam JM. A review of isolated third nerve palsy without subarachnoid hemorrhage using computed tomographic angiography as the first line of investigation. Clin Neurol Neurosurg 2004; 107: 27-31.

4. Ahn JY, Han IB, Yoon PH, Kim SH, Kim S, Joo JY. Clipping $v$ s coiling of posterior communicating artery aneurysm with third nerve palsy. Neurology 2006; 66: 121-123.

5. La Mantia L, Curone M, Rapoport AM, Bussone G; International Headache Society. Tolosa-Hunt syndrome: critical literature review based on IHS 2004 criteria. Cephalalgia 2006; 26: 772-781. 